\title{
The Complexity of Modular Graph Automorphism
}

\author{
V. Arvind * \\ Institute of Mathematical Sciences University of Illinois at Chicago \\ A. Lozano \\ Universitat Politècnica de Catalunya
}

\begin{abstract}
Motivated by the question of the relative complexities of the Graph Isomorphism and the Graph Automorphism problems, we define and study the modular graph automorphism problems. These are the decision problems $\bmod _{k}$-GA which consist, for each $k>1$, of deciding whether the number of automorphisms of a graph is divisible by $k$. The $\bmod _{k}$-GA problems all turn out to be intermediate in difficulty between Graph Automorphism and Graph Isomorphism.

We define an appropriate search version of $\bmod _{k}$-GA and design an algorithm that polynomial-time reduces the $\bmod _{k}$-GA search problem to the decision problem. Combining this algorithm with an IP protocol, we obtain a randomized polynomial-time checker for $\bmod _{k}$-GA, for all $k>1$.
\end{abstract}

\section{Introduction}

The Graph Isomorphism problem (GI) consists of determining whether two graphs are isomorphic. It is well known that GI is in NP, but despite decades of study by mathematicians and computer scientists, it is not known whether GI is in P or whether GI is NPcomplete. Many researchers conjecture that GI's complexity lies somewhere between $\mathrm{P}$ and NP-complete. Related to GI are several other decision problems (some graph-theoretic and others group-theoretic in nature) that are similarly not known to be in $\mathrm{P}$ or NP-complete. One such problem which is closely related to GI is Graph Automorphism (GA): Deciding

\footnotetext{
${ }^{*}$ Madras 600113, India (email: arvind@imsc.ernet.in)

${ }^{\dagger}$ Research performed at the University of Maryland and Yale University. Address: Dept. of Electrical Engineering and Computer Science, University of Illinois at Chicago, $851 \mathrm{~S}$ Morgan St, Chicago IL 60607-7053, USA. Supported in part by the National Science Foundation under grants CCR-8958528, CCR9700417, and CCR-9796317 and by NASA under grant NAG 52895. Email: beigel@eecs.uic.edu. Web: http://www.eecs.uic.edu/ 'beigel/

${ }_{\ddagger}^{\ddagger}$ L.S.I. Dept., Campus Nord, Mòdul C6, Jordi Girona 1-3, E08034-Barcelona, Catalonia, E. U. (email: antoni@lsi.upc.es). Supported in part by the E.U. through ESPRIT project (no. LTR 20244, ALCOM-IT) and by DGICYT (Koala project, no. PB95-0787), and CICYT (no. TIC97-1475-CE). Work completed while visiting the first author at the Institute of Mathematical Sciences.
} 
whether a graph has a nontrivial automorphism. Regarding the relative complexities of GA and GI, it is known that GA is polynomial-time many-one reducible to GI. On the other hand, GI is not known to be even polynomial-time Turing reducible to GA (see [10] for these and related results). However, in [12] it is shown that GI is polynomial-time reducible to the problem of computing the number of automorphisms of a graph.

The notion of program checking was introduced by Blum and Kannan [3] as an algorithmic alternative to program verification. Since then the design of efficient checkers for various computational problems has rapidly grown into a discipline of algorithm design $[3,4]$. One of the first program checkers in [3] was a randomized polynomial-time checker for GI. It is an outstanding open question in the area if NP-complete problems have efficient program checkers. This can be construed as another evidence that GI is not NP-complete. Later, in [11] it was shown that GA has a nonadaptive checker. In other words, the checker can make all its queries to the program in parallel, hence enabling it to be fast in parallel (in NC, to be precise). It is an open question whether GI too has a nonadaptive checker, and the apparent bottleneck here is that the search problem for GI is not known to be polynomialtime truth-table reducible to the decision problem for GI (i.e. the reduction is nonadaptive: it uses only parallel queries).

Thus, a natural next step in investigating the relationship between GI and GA is to consider exactly how much we need to know about the number of automorphisms of a graph in order to solve the Graph Isomorphism problem. This motivates us to define and study modular graph automorphism problems. Let $A u t(G)$ denote the automorphism group of the graph $G$.

Definition 1 For any $k$, let $\bmod _{k}-\mathrm{GA}=\{G:|A u t(G)| \equiv 0 \quad(\bmod k)\}$.

We show in Theorems 4 and 5 that for any $k>1$, GA $\leq_{m}^{p} \bmod _{k}$-GA $\leq_{m}^{p}$ GI; thus the $\bmod _{k}$-GA problems are intermediate in difficulty between GA and GI. It is an open question whether any of the $\bmod _{k}$-GA problems is polynomial-time equivalent to GA or GI. We conjecture that $\bmod _{k}$-GA is not polynomial-time equivalent to GA or GI, for any $k>1$. An evidence that some of the $\bmod _{k}$-GA problems could be actually harder than GA is our observation that Tournament Isomorphism (graph isomorphism for tournament graphs) is many-one reducible to $\bmod _{2}-\mathrm{GA}$. This follows from the fact that the automorphism group of any tournament is of odd size [10], which in turn implies that two tournaments are isomorphic iff the automorphism group of their disjoint union contains an order-two permutation (which must switch the two graphs).

The layout of the paper is as follows. Section 2 contains the preliminaries. In Section 3, we prove that the $\bmod _{k}$-GA problems are located between GA and GI. In Section 4, we show that search is polynomial-time Turing equivalent to decision for $\bmod _{k}$-GA, and in Section 5 we use this result in combination with an IP protocol for $\overline{\bmod _{p} \text {-GA }}$ to obtain an efficient program checker for $\bmod _{k}$-GA. Notice that although both GA and GI have program checkers (shown in [11] and [3] resp.) and $\bmod _{k}$-GA is intermediate in complexity, it does not necessarily imply that $\bmod _{k}$-GA has a program checker [3]. 


\section{Preliminaries}

In this paper by a graph we mean a finite directed graph ${ }^{1}$ (see for example [8] or any other standard text on graph theory for basic definitions). For a graph $G$, let $V(G)$ denote its vertex set and $E(G)$ denote its edge set. A permutation $\pi$ on the vertex set $V(G)$ of a graph $G$ is an automorphism of $G$ if $(u, v) \in E(G) \Longleftrightarrow(\pi(u), \pi(v)) \in E(G)$. The set of automorphisms $A u t(G)$, of a graph $G$, is a subgroup of the permutation group on $V(G)$. The identity automorphism of any graph will be denoted by id.

Let $X$ be a list of vertices in $V(G)$ for a given graph $G$. By $G_{[X]}$ we mean the graph $G$ with distinct labels attached to the vertices in $X$. Given two lists of vertices $X, Y \subseteq V(G)$, the graphs $G_{[X]}$ and $G_{[Y]}$ have the same labels in vertices occupying the same relative positions in $X$ and $Y$. It is not hard to see that in $G_{[X]}$ vertices of $X$ are pointwise fixed in any automorphism ${ }^{2}$. Thus $A u t\left(G_{[X]}\right)$ is isomorphic to the subgroup of $A u t(G)$ which pointwise fixes the vertices in $X$. Furthermore, given an automorphism of $A u t\left(G_{[X]}\right)$ the corresponding automorphism of $A u t(G)$ can be efficiently (i.e. in polynomial time) constructed.

Definition 2 Let $G_{1}, \ldots, G_{n}$ be $n$ graphs.

- Let $P_{n}$ be a directed simple path of $n$ new vertices $v_{1}, v_{2}, \ldots, v_{n}$, where each vertex $v_{i}$ is labeled with a single label $l$. The graph Path $\left(G_{1}, \ldots, G_{n}\right)$ is obtained by taking one copy of each of the graphs $G_{1}, \ldots, G_{n}$ and, for $1 \leq i \leq n$, attaching all the vertices of $G_{i}$ to $v_{i}$.

- Let $C_{n}$ denote the directed simple cycle on $n$ new vertices $v_{1}, v_{2}, \ldots, v_{n}$, with each vertex $v_{i}, 1 \leq i \leq n$, labeled with a single label l. The graph Cycle $\left(G_{1}, \ldots, G_{n}\right)$ is obtained by taking one copy of each of the graphs $G_{1}, \ldots, G_{n}$ and, for $1 \leq i \leq n$, attaching all the vertices of $G_{i}$ to $v_{i}$.

In both $\operatorname{Path}\left(G_{1}, \ldots, G_{n}\right)$ and $\operatorname{Cycle}\left(G_{1}, \ldots, G_{n}\right)$, since the new vertices $v_{1}, v_{2}, \ldots, v_{n}$ are labeled with $l$, any automorphism of these graphs must map the set $\left\{v_{1}, v_{2}, \ldots, v_{n}\right\}$ onto itself. Consequently, any automorphism of $\operatorname{Path}\left(G_{1}, \ldots, G_{n}\right)\left(\operatorname{Cycle}\left(G_{1}, \ldots, G_{n}\right)\right)$ when restricted to $\left\{v_{1}, v_{2}, \ldots, v_{n}\right\}$ is an automorphism of $P_{n}\left(C_{n}\right)$ This means that an automorphism of $\operatorname{Path}\left(G_{1}, \ldots, G_{n}\right)$ cannot permute the copies of $G_{1}, \ldots, G_{n}$, while an automorphism of Cycle $\left(G_{1}, \ldots, G_{n}\right)$ can permute them but only along the cycle $C_{n}$.

The reducibilities discussed in this paper are the standard polynomial-time Turing and many-one reducibilities. Formal definitions of these and other standard notions in complexity theory can be found in $[2,1]$.

We finish this section with some complexity-theoretic concepts which will be used later. A set $A \subseteq \Sigma^{*}$ is a $d$-cylinder if there is an FP function OR that takes a list of strings $x_{1}, x_{2}, \ldots, x_{m}$ as argument and produces a string $y$ such that

$$
\mathrm{OR}\left(x_{1}, x_{2}, \ldots, x_{m}\right)=y \in A \Longleftrightarrow \exists i: 1 \leq i \leq m: x_{i} \in A
$$

\footnotetext{
${ }^{1}$ In this paper we consider the problems GI, GA, and $\bmod _{k^{-}}$GA on directed graphs. However, all results of this paper hold for these problems on undirected graphs as well.

${ }^{2}$ Each label can be implemented with a graph gadget like a long path such that the overall size of the graph is still polynomially bounded. See, e.g. [10].
} 
Similarly, a set $A \subseteq \Sigma^{*}$ is a $c$-cylinder if there is an FP function AND that takes a list of strings $x_{1}, x_{2}, \ldots, x_{m}$ as argument and produces a string $y$ such that

$$
\operatorname{AND}\left(x_{1}, x_{2}, \ldots, x_{m}\right)=y \in A \Longleftrightarrow \forall i: 1 \leq i \leq m: x_{i} \in A
$$

Now, we recall that GI satisfies both properties ${ }^{3}$.

Proposition $3[5,11]$ GI is a d-cylinder and a c-cylinder.

The relative complexity of decision and search for NP problems is well studied [2, 1]. For instance, it is known that search and decision are polynomial-time Turing equivalent for all NP-complete problems. In particular, we recall that for GI, search is polynomial-time Turing reducible to decision [13] whereas for GA a stronger result holds: search is nonadaptively polynomial-time reducible to decision [11].

\section{Locating the $\bmod _{k}$-GA Problems}

We show in this section that $\bmod _{k}$-GA is located between GA and GI, for all $k>1$.

Theorem 4 For all $k>1, \mathrm{GA} \leq_{m}^{p} \bmod _{k^{-}} \mathrm{GA}$.

Proof. Given a graph $G$, we define for every $i, j$ with $1 \leq i<j \leq n$, the graph $H_{i, j}=$ $\operatorname{Cycle}\left(G_{[\{i\}]}, G_{[\{j\}]}, \ldots, G_{[\{j\}]}\right)$ which contains one copy of $G_{[\{i\}]}$ and $k-1$ copies of $G_{[\{j\}]}$. Further, let $H$ be obtained by applying the Path operator to all the graphs $H_{i, j}$ with $1 \leq$ $i<j \leq n$. We claim that $G$ has a nontrivial automorphism if and only if $H$ is in mod $_{k}$-GA.

Suppose that $G$ has a nontrivial automorphism $\varphi$. There exist two vertices $i$ and $j$ such that $\varphi(i)=j$. Notice that $H_{i, j}$ has the following nontrivial automorphism $\alpha$ that cyclically permutes the $k$ graphs in $\operatorname{Cycle}\left(G_{[\{i\}]}, G_{[\{j\}]}, \ldots, G_{[\{j\}]}\right)$ as follows. The automorphism $\alpha$ maps the first graph $G_{[\{i\}]}$ to $G_{[\{j\}]}$ by $\varphi$. It maps each of the first $k-2$ copies of $G_{[\{j\}]}$ to the next copy of $G_{[\{j\}]}$ by the identity automorphism. Finally, $\alpha$ maps the last copy of $G_{[\{j\}]}$ back to $G_{[\{i\}]}$ by the automorphism $\varphi^{-1}$.

The order of $\alpha$ is $k$ since the vertices in $H_{i, j}$ are moved in a cyclic way through the different $k$ subgraphs. In fact, the permutation $\alpha$ is a product of a bunch of $k$-cycles. Thus $H_{i, j} \in \bmod _{k}$-GA. Since $|A u t(H)|=\prod_{1 \leq i<j<n}\left|A u t\left(H_{i, j}\right)\right|$, it follows that $H \in \bmod _{k}$-GA.

For the converse, assume that $H \in \bmod _{k}$-GA. Then, $H$ has a nontrivial automorphism, say, $\alpha$. Notice that $\alpha$ must induce an automorphism $\beta$ in one of its subgraphs $H_{i, j}$. Since $H_{i, j}=\operatorname{Cycle}\left(G_{[\{i\}]}, G_{[\{j\}]}, \ldots, G_{[\{j\}]}\right)$, there are two possibilities: either $\beta$ induces a nontrivial automorphism of $G_{[\{i\}]}$ or $G_{[\{j\}]}$, or else $\beta$ maps the copy of $G_{[\{i\}]}$ to some copy of $G_{[\{j\}]}$. In either case, it is clear that we get a nontrivial automorphism of $G$.

Mathon [12] has shown that $|A u t(G)|$ is polynomial-time computable with GI as oracle. From this it easily follows that $\bmod _{k}-\mathrm{GA} \leq_{T}^{p} \mathrm{GI}$. In the next theorem, we strengthen this to a $\leq_{m}^{p}$-reduction using some permutation group theory.

\footnotetext{
${ }^{3}$ Elsewhere in the literature, e.g. [10], these properties are called OR and AND functions respectively.
} 
Theorem 5 For all $k>1, \bmod _{k}-\mathrm{GA} \leq_{m}^{p} \mathrm{GI}$.

We need a couple of definitions and group-theoretic lemmas before we prove Theorem 5 . Let $A$ be a subgroup of $S_{n}$ and let $[n]$ denote the set $\{1,2, \ldots, n\}$. A subset $X \subseteq[n]$ is $A$-invariant if $g(X)=X$ for all $g \in A$. If $X \subseteq[n]$ is $A$-invariant then consider the action of $A$ restricted to $X$. This gives rise to a subgroup of the symmetric group $S_{X}$, which we denote by $A^{X}$. A useful property that is obvious is that $\left|A^{X}\right| \leq|A|$, for all $A$-invariant sets $X$.

Lemma 6 Let $A$ be a subgroup of $S_{n}$ s.t. $|A|=m$. Then there exists an $A$-invariant subset $X \subseteq[n]$ with $|X| \leq m \log m$, such that $A$ is isomorphic to $A^{X}$.

Proof. Consider the following procedure for constructing the set $X$ :

$X \leftarrow \emptyset$

while $\exists i \notin X:\left|A^{X}\right|<\left|A^{X \cup A(i)}\right|$ do

${ }^{*} A(i)$ denotes the orbit of $i$ under $A * /$

\{

Pick such an $i$;

$X \leftarrow X \cup A(i)$

\}

First we claim that, as a loop invariant, $X$ is always an $A$-invariant subset of $[n]$. To see this, notice that it holds at the beginning when $X$ is empty, and if $X$ is $A$-invariant then so is $X \cup A(i)$ since we are including an entire $A$-orbit in the set.

Next, suppose $X$ is $A$-invariant and $i \notin X$ is some index. Consider the mapping $\varphi$ from $A^{X \cup A(i)}$ to $A^{X}$ which maps an element of $A^{X \cup A(i)}$ to its restriction to $X$. Since $X$ is $A$-invariant, it is easy to verify that $\varphi$ is a surjective homomorphism from $A^{X \cup A(i)}$ to $A^{X}$. It follows that $\left|A^{X}\right|$ divides $\left|A^{X \cup A(i)}\right|$. Suppose now, at some stage of the while loop, $i$ is an index such that $\left|A^{X}\right|<\left|A^{X \cup A(i)}\right|$. Then it must hold that $2\left|A^{X}\right| \leq\left|A^{X \cup A(i)}\right|$. Thus we have argued that every time $X$ increases by including an orbit $A(i)$ in it, the size of the group $A^{X}$ increases by at least a factor of 2. Thus the assignment $X \leftarrow X \cup A(i)$ is executed at most $\log m$ times, implying also that the procedure must stop. Since the size of any orbit $A(i)$ is bounded by $|A|$, it follows that the procedure stops with an $A$-invariant set $X$ such that $|X| \leq m \log m$. Let $X$ be the set computed when the while-loop is exited. To complete the proof we must show that $A^{X}$ is isomorphic to $A$. Consider the canonical surjective homomorphism $\psi$ from $A$ to $A^{X}$, which maps a given element of $A$ to its corresponding restriction to $X$. To show that this homomorphism is an isomorphism we only need to argue that $\operatorname{Ker}(\psi)$ is (id). Suppose $g \in \operatorname{Ker}(\psi)$ is a nontrivial element. Then there is $i \notin X$ such that $g(i) \neq i$. This in turn implies that the surjective homomorphism $\varphi$ from $A^{X \cup A(i)}$ to $A^{X}$ which maps an element of $A^{X \cup A(i)}$ to its restriction to $X$, has a nontrivial kernel with $g \in \operatorname{Ker}(\varphi)$. Consequently, $\left|A^{X}\right|<\left|A^{X \cup A(i)}\right|$. Thus, both $X$ and $i$ satisfy the while-loop condition contradicting the fact that the while loop has terminated. This completes the proof of this lemma. 
Lemma 7 Let $A$ be a finite group. Let $X=\left\{a_{1}, a_{2}, \ldots, a_{t}\right\}$ and $Y=\left\{b_{1}, b_{2}, \ldots, b_{t}\right\}$ be two subsets of $A$ such that $\langle X\rangle \cap\langle Y\rangle=$ (id) and $a_{i} b_{j}=b_{j} a_{i}$, for $1 \leq i, j \leq t$. Then $|\langle X\rangle|$ divides the order of the group $\left\langle\left\{a_{i} b_{i}: 1 \leq i \leq t\right\}\right\rangle$.

Proof. Let $H$ denote the subgroup of $A$ generated by $\left\{a_{i} b_{i} \mid 1 \leq i \leq t\right\}, K$ denote the subgroup of $A$ generated by $\left\{a_{i} \mid 1 \leq i \leq t\right\}$, and $L$ denote the subgroup of $A$ generated by $\left\{b_{i} \mid 1 \leq i \leq t\right\}$. Notice that, since $a_{i} b_{j}=b_{j} a_{i}$, for $1 \leq i, j \leq t$, we have $K L=L K$ and therefore the set $K L$ is actually a subgroup of $A$. Next, notice that, by definition of $H$, any $x \in H$ can be written as a product of elements from the generator set $\left\{a_{i} b_{i} \mid 1 \leq i \leq t\right\}$. Using $a_{i} b_{j}=b_{j} a_{i}$, for $1 \leq i, j \leq t$ as a rewrite rule, this product of generators expressing $x$ can be rewritten as $a y$, where $a \in K$ and $y \in L$. It follows that $H \subseteq K L$. Consider the following map $\psi$ from the group $H$ to the group $K$ defined as follows:

$$
\forall x \in H: \psi(x)=a \text { where } x=a y, \text { with } a \in K \text { and } y \in L
$$

We claim that $\psi$ is a well-defined surjective homomorphism from $H$ to $K$. We first show that $\psi$ is well-defined. Suppose there are two distinct elements $a, a^{\prime} \in K$ such that $x=a y=a^{\prime} y^{\prime}$ for elements $y, y^{\prime} \in L$. This implies, by cancelation laws, that $a^{-1} a^{\prime}=y y^{\prime-1}$, which belongs to both $K$ and $L$. Since $K \cap L=$ (id), we have $a=a^{\prime}$. Thus $\psi$ is well-defined. To see that $\psi$ is a homomorphism is routine: we can easily check that $\psi\left(x x^{\prime}\right)=\psi(x) \psi\left(x^{\prime}\right)$ and that $\psi\left(x^{-1}\right)=(\psi(x))^{-1}$ hold using the rewrite rules $a_{i} b_{j}=b_{j} a_{i}$, for $1 \leq i, j \leq t$. To see that $\psi$ is surjective, let $a \in K$ be any element. We can express $a$ as a product $\Pi_{1 \leq r \leq m} a_{i_{r}}$ for indices $i_{r} \in[t]$. Consider the element $x=\Pi_{1 \leq r \leq m} a_{i_{r}} b_{i_{r}} \in H$. It is easy to see that $\bar{\psi}(x)=a$.

Thus by the fundamental theorem of homomorphisms it follows that $H / \operatorname{Ker}(\psi)$ is isomorphic to $K$. Therefore, $|H / \operatorname{Ker}(\psi)|=|K|$. It follows that $|K|$ divides $|H|$ which proves the lemma.

\section{Proof of Theorem 5}

First, we argue that it suffices to show that $\bmod _{p^{l}}$ GA $\leq_{m}^{p}$ GI for all prime $p$ and $l>0$. To see this, let $\prod_{1 \leq j \leq r} p_{j}^{l_{j}}$ be the prime factorization of $k$. Clearly, a graph $G \in \bmod _{k}$-GA iff $G \in \bigcap_{1 \leq j \leq r} \bmod _{p_{j}^{l_{j}}}$ GA. Thus, if $\bmod _{p_{j}^{l_{j}}}-\mathrm{GA} \leq_{m}^{p}$ GI for $1 \leq j \leq r$, it follows that $\bmod _{k}$-GA $\leq_{m}^{p}$ GI, since GI is a c-cylinder.

We first prove a useful group-theoretic claim. Let $G$ be a graph on $n$ vertices and $f$ be a partial permutation on [n] (i.e. $f$ is defined on a subset of the domain $[n]$ and can be extended to a permutation in $S_{n}$ ). Then we call $f$ a partial automorphism of $G$ if $f$ can be extended to an automorphism of $G$.

Claim. Let $p$ be a fixed prime and $l>0 . A$ graph $G$ on $n$ vertices is in $\bmod _{p^{i}} \mathrm{GA}$ if and only if there exist a set $X \subseteq[n]$ with $|X| \leq p^{l}\left(\log p^{l}\right)$ and a subgroup $K=\left\{a_{1}, a_{2}, \ldots, a_{p^{l}}\right\}$ of $S_{X}$ such that each $a_{i} \in K$ is a partial automorphism of $G$.

Proof. Let $G \in \bmod _{p^{1}}$-GA be an $n$ vertex graph. Since $p^{l}$ divides $|A u t(G)|$, by Sylow's theorem $\operatorname{Aut}(G)$ has a subgroup $A$ of size $p^{l}$. By Lemma 6 there is an $A$-invariant set $X \subseteq[n]$ with $|X| \leq p^{l}\left(\log p^{l}\right)$, such that $A^{X}$ is isomorphic to $A$. Let $A^{X}=\left\{a_{1}, a_{2}, \ldots, a_{p^{l}}\right\}$. Furthermore, it also follows that $A^{X}$ is a subgroup of $S_{X}$ where each $a_{i} \in A^{X}$ is a partial 
automorphism of $G$. Conversely, suppose there is $X \subseteq[n]$ with $|X| \leq p^{l}\left(\log p^{l}\right)$ and a subgroup $K=\left\{a_{1}, a_{2}, \ldots, a_{p^{l}}\right\}$ of $S_{X}$ where each $a_{i} \in K$ is a partial automorphism of $G$. Then for each $i$ with $1 \leq i \leq p^{l}$, there is a $b_{i} \in S_{[n]-X}$ such that $a_{i} b_{i} \in A u t(G)$. We can now apply Lemma 7 to the elements $\left\{a_{i}\right\}_{1 \leq i \leq p^{l}}$ and $\left\{b_{i}\right\}_{1 \leq i \leq p^{l}}$, since the required conditions are fulfilled. Consequently, $\left|\left\langle\left\{a_{i} b_{i}: 1 \leq i \leq p^{l}\right\}\right\rangle\right|$ is divisible by $p^{l}$. Since $\left\langle\left\{a_{i} b_{i}: 1 \leq i \leq p^{l}\right\}\right\rangle$ is a subgroup of $A u t(G)$, it follows that $p^{l}$ divides $|A u t(G)|$.

Now, note that the language $B=\{(G, f): f$ is a partial automorphism of the graph $G\}$ is $\leq_{m}^{p}$-reducible to GI (for details see [10]). We will give a truth-table reduction from $\bmod _{p^{l}}$-GA to $B$, where the truth-table is a disjunction of conjunctions. Since the language $B$ is $\leq_{m}^{p}$-reducible to GI and since GI is both a c-cylinder and a d-cylinder, it follows that $\bmod _{p^{l}}$-GA is $\leq_{m}^{p}$-reducible to GI. We describe below the said reduction of $\bmod _{p^{l}}$-GA to $B$ as a logical expression, which is easily seen to describe a disjunction-of-conjunctions kind of truth-table reduction:

$$
\begin{aligned}
G \in \bmod _{p^{l}-\mathrm{GA}} \Longleftrightarrow \quad & \left(\exists X \subseteq[n]:|X| \leq p^{l} \log p^{l}\right) \\
& \left(\exists \operatorname{subgroup} K<S_{X}:|K|=p^{l}\right)(\forall a \in K)[(G, a) \in B]
\end{aligned}
$$

This completes the proof of Theorem 5 .

\section{Computing Solutions for $\bmod _{k^{-}}$GA Instances}

The goal of this section is to design a polynomial-time algorithm that reduces the search problem for $\bmod _{k}$-GA to the decision problem. Consider $\bmod _{k}$-GA for an arbitrary $k>1$. Notice that if the prime factorization of $k$ is $\prod_{1 \leq i \leq m} p_{i}^{e_{i}}$, then the natural NP witness of the membership of a graph $G$ in $\bmod _{k}$-GA is a collection of $m$ subgroups $\left\{A_{1}, A_{2}, \ldots, A_{m}\right\}$ of $\operatorname{Aut}(G)$ where, for each $i, A_{i}$ is of order $p_{i}^{e_{i}}$, and $A_{i}$ is listed as a set of permutations. We consider such a witness as a solution for $G$ for the $\bmod _{k}$-GA search problem and we design a polynomial-time algorithm that computes this witness for any given instance of $\bmod _{k}$-GA with oracle access to the $\bmod _{k}$-GA decision problem.

In the following lemma we introduce one of the two last graph gadgets which we will need in order to prove the main result of this section.

Lemma 8 Given $t$ graphs $G_{1}, G_{2}, \ldots, G_{t}$, each with n nodes, we can construct in polynomial time a new graph Paste $\left(G_{1}, G_{2}, \ldots, G_{t}\right)$ such that the following properties hold.

1. A permutation $\psi \in S_{n}$ is an automorphism of Paste $\left(G_{1}, G_{2}, \ldots, G_{t}\right)$ iff there is a permutation $\pi \in \bigcap_{1 \leq i \leq t} A u t\left(G_{i}\right)$ such that $\psi$ restricted to $G_{i}$ is $\pi$, for $1 \leq i \leq t$.

2. Let $p$ be a prime. Paste $\left(G_{1}, G_{2}, \ldots, G_{t}\right)$ has an automorphism of order $p$ iff there is an order-p permutation $\pi \in \bigcap_{1 \leq i \leq t} A u t\left(G_{i}\right)$.

3. Given $\psi \in \operatorname{Aut}\left(\operatorname{Paste}\left(G_{1}, G_{2}, \ldots, G_{t}\right)\right)$ we can in polynomial time construct the corresponding $\pi \in \bigcap_{1 \leq i \leq t} A u t\left(G_{i}\right)$. 
Proof. Notice that parts 2 and 3 of the lemma are both direct consequences of part 1 . Thus it suffices to prove the first part. The graph Paste $\left(G_{1}, G_{2}, \ldots, G_{t}\right)$ basically consists of one copy of each of $G_{1}, G_{2}, \ldots, G_{t}$. Furthermore, for $1 \leq i \leq t$ we color the nodes of the copy of $G_{i}$ using color $C_{i}$. This forces every automorphism of the new graph to map the copy of $G_{i}$ to itself. Next, we use $n$ distinct labeling nodes $L_{j}, 1 \leq j \leq n$, as follows: from the $j$ th node of every graph $G_{i}$ put a long path of some fixed length $N$ to node $L_{j}$. This ensures that for any automorphism of Paste $\left(G_{1}, G_{2}, \ldots, G_{t}\right)$, if node $j_{1}$ is mapped to node $j_{2}$ in $G_{i}$ then $j_{1}$ gets mapped to the node $j_{2}$ also for each $G_{i^{\prime}}, i^{\prime} \neq i$. This construction guarantees the following: given an automorphism $\psi$ of $\operatorname{Paste}\left(G_{1}, G_{2}, \ldots, G_{t}\right)$, there is a permutation $\pi \in \bigcap_{1 \leq i \leq t} \operatorname{Aut}\left(G_{i}\right)$ such that $\psi$ restricted to $G_{i}$ is $\pi$, for $1 \leq i \leq t$. This proves the lemma.

Before we proceed we need to recall a definition.

Definition 9 [9] Let $\pi \in S_{n}$ be a permutation. The cycle graph of $\pi$ is the directed graph $G=([n], E)$, where $(i, j) \in E$ iff $\pi(i)=j$.

We next recall a lemma from [9].

Lemma 10 [9] If $G$ is the cycle graph of $\pi \in S_{n}$ then Aut $(G)$ is precisely the set of all permutations in $S_{n}$ that commute with $\pi$.

The second graph gadget needed is the following.

Lemma 11 Let $G$ be a graph on $n$ nodes and $S=\left\{g_{1}, g_{2}, \ldots, g_{t}\right\} \subseteq S_{n}$ be a set of permutations. Further let $\mathcal{C}=\left\{C_{1}, C_{2}, \ldots, C_{s}\right\} \subseteq S_{n}$ be a set of pairwise disjoint cycles, $p$ be a fixed prime, and $\tau$ be a permutation on $[t]$. Then we can compute in time polynomial in $n$ a graph $\operatorname{Comb}(\tau, G, S, \mathcal{C}, p)$ such that $\operatorname{Comb}(\tau, G, S, \mathcal{C}, p) \in \bmod _{p}$-GA iff one of the following holds.

1. G has a nontrivial automorphism $\pi$ of order $p$ such that $\pi g_{i} \pi^{-1}=g_{i}$, for $1 \leq i \leq t$, and such that $\pi(x)=x$ for all $x \in \bigcup_{1 \leq i \leq s} C_{i}$.

2. $G$ has a nontrivial automorphism $\pi$ such that $C_{1}, C_{2}, \ldots, C_{s}$ are cycles of $\pi$ and such that $\pi g_{i} \pi^{-1}=g_{\tau(i)}$, for $1 \leq i \leq t$.

Proof. Let the composition $C_{1} C_{2} \cdots C_{s}$ of the cycles of $\mathcal{C}$ be denoted by $\psi \in S_{n}$. Further, let $G^{\prime}$ denote the graph obtained from $G$ by coloring each node $x \in \bigcup_{1 \leq i \leq s} C_{i}$ with a distinct color $n_{x}$. Similarly, let $G^{\prime \prime}$ denote the graph obtained from $G$ by coloring each node $\psi(x) \in$ $\bigcup_{1 \leq i \leq s} C_{i}$ with the color $n_{x}$, for each $x$ (where $n_{x}$ is used to color node $x$ in $G^{\prime}$ ).

Now, let $H=\operatorname{Paste}\left(G^{\prime}, G_{1}, G_{2}, \ldots, G_{t}\right)$, where $G_{i}$ is the cycle graph of $g_{i}$, for $1 \leq i \leq t$. Similarly, let $K=\operatorname{Paste}\left(G^{\prime \prime}, G_{\tau(1)}, G_{\tau(2)}, \ldots, G_{\tau(t)}\right)$. Finally, we put one copy of $H$ and $p-1$ copies of $K$ together to build the graph $C y c l e(H, K, \ldots, K)$ (in which we have $p-1$ copies of $K)$. This graph $C y c l e(H, K, \ldots, K)$ is defined to be $C o m b(\tau, G, S, \mathcal{C}, p)$.

Suppose $\operatorname{Comb}(\tau, G, S, \mathcal{C}, p) \in \bmod _{p}-\mathrm{GA}$. Now, suppose the first of the above two properties does not hold for $G$. We will prove that the second property must hold. Let $\rho$ be an order-p automorphism of the graph $\operatorname{Comb}(\tau, G, S, \mathcal{C}, p)$. Since the first property does not 
hold, notice that the order- $p$ automorphism $\rho$ of the graph $C y c l e(H, K, \ldots, K)$ cannot map $H$ to itself and each copy of $K$ to itself. Therefore, since $p$ is prime, $\rho$ must permute the $p$ graphs in the list $(H, K, \ldots, K)$ by a $p$-cycle. More precisely, $\rho$ can be seen as a $p$-tuple $\left(\rho_{1}, \rho_{2}, \ldots, \rho_{p}\right)$ of permutations $\rho_{i} \in S_{n}, 1 \leq i \leq p$, where $\rho_{1}$ maps the copy of $H$ into a copy of $K$, and the permutations $\rho_{2}, \ldots, \rho_{p-1}$ map a copy of $K$ into a fresh copy of $K$, and finally $\rho_{p}$ maps a copy of $K$ back to $H$. Recall that $H=\operatorname{Paste}\left(G^{\prime}, G_{1}, G_{2}, \ldots, G_{t}\right)$ and $K=\operatorname{Paste}\left(G^{\prime \prime}, G_{\tau(1)}, G_{\tau(2)}, \ldots, G_{\tau(t)}\right)$, and observe that $\rho_{1}$, which maps $H$ to $K$, is forced due to the color labels to map $G^{\prime}$ to $G^{\prime \prime}$ and to map $G_{i}$ to $G_{\tau(i)}$ for each $i, 1 \leq i \leq t$. Thus, $\rho_{1}$ is an automorphism of $G$ that has $C_{1}, C_{2}, \ldots, C_{s}$ as its cycles, and $g_{i} \rho_{1} g_{i}^{-1}=g_{\tau(i)}$, for $1 \leq i \leq t$ as claimed by the second property.

For the converse implication, suppose the first property holds. Let $\pi$ be an order- $p$ antomorphism of $G$ satisfying the first property. Consider the permutation $\nu$ of the nodes of the graph $\operatorname{Comb}(\tau, G, S, \mathcal{C}, p)$, where the copy of $H$ and each copy of $K$ is mapped to itself under $\pi$. Clearly, $\nu$ is an order-p automorphism of $\operatorname{Comb}(\tau, G, S, \mathcal{C}, p)$. Next, suppose that the first property fails and the second property holds. Again, let $\pi$ denote the automorphism of $G$ satisfying the second property. Consider the permutation $\mu$ of the nodes of $\operatorname{Comb}(\tau, G, S, \mathcal{C}, p)$, which maps the copy of $H$ into the first copy of $K$ according to $\pi$, and then successively maps the first $p-2$ copies of $K$ by the identity permutation into the corresponding next copy of $K$ in the cyclic order, and finally maps the last copy of $K$ to $H$ according to $\pi^{-1}$. Observe that the permutation $\mu$ is in fact a product of disjoint $p$-cycles: the $p$-cycles are the orbits of vertices of $H$. It follows that $\mu$ is an order- $p$ automorphism of $\operatorname{Comb}(\tau, G, S, \mathcal{C}, p)$.

The next theorem is the main result of this section. Its proof draws on group-theoretic results concerning $p$-groups.

Theorem 12 For any prime $p$, there is a polynomial-time algorithm $\mathcal{A}_{k}$ with mod, ${ }_{p}$ GA as oracle such that given a graph $G \in \bmod _{p^{k}} \mathrm{GA}$ as input, the algorithm $\mathcal{A}_{k}$ lists out the elements of an order-p $p^{k}$ subgroup of $A u t(G)$.

We will prove Theorem 12 by induction on $k$. We first take care of the base case (when $k=1$ ) in the following lemma.

Lemma 13 For any prime $p$, there is a polynomial-time algorithm $\mathcal{A}_{1}$ with mod $_{p}$-GA as oracle such that given a graph $G \in \bmod _{p}$-GA as input, the algorithm $\mathcal{A}_{1}$ outputs a cyclic group of order $p$ contained in $\operatorname{Aut}(G)$.

Proof. For any list of vertices $X=\left\{i_{1}, \ldots, i_{m}\right\}$, let $r(X)$ be a right shift of $X$, this is $r(X)=\left\{i_{m}, i_{1}, \ldots, i_{m-1}\right\}$. Consider the following algorithm, which computes an order- $p$ automorphism of an input graph $G \in \bmod _{p}-\mathrm{GA}$.

\section{Algorithm $\mathcal{A}_{1}$ :}

input $G$;

if $G \notin \bmod _{p}$ GA then stop;

$X \leftarrow \emptyset$; 


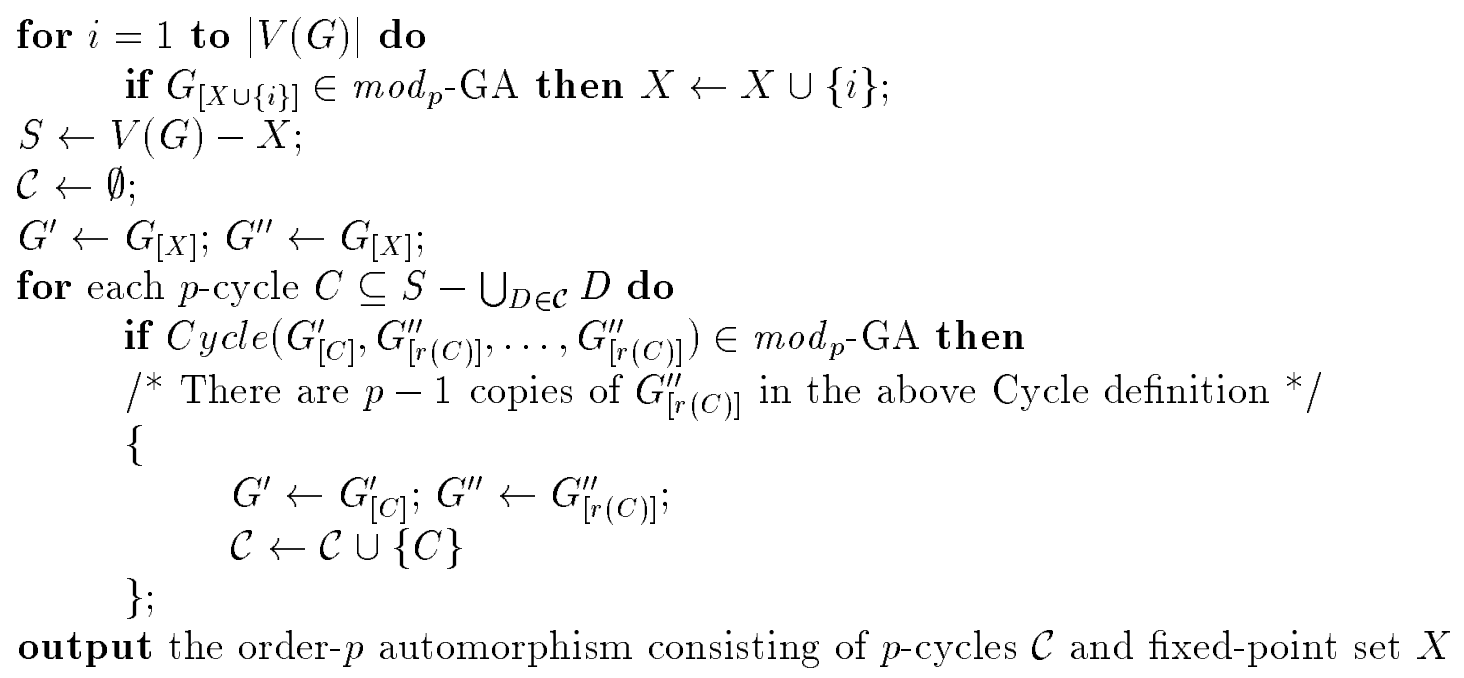

We now prove the correctness of the above algorithm. Notice that the first for-loop takes $G \in \bmod _{p}$-GA as input and computes the graph $G_{[X]} \in \bmod _{p}$-GA with $X$ as its set of fixed points (such that no more points can be fixed preserving membership in $\bmod _{p}-\mathrm{GA}$ ). We have to show that when the algorithm stops it outputs an order- $p$ automorphism which has $\mathcal{C}$ as its collection of $p$-cycles and $X$ as its fixed-point set. To begin with, notice that any order- $p$ automorphism with $X$ as its fixed-point set is a product of disjoint $p$-cycles and 1-cycles (corresponding to elements of $X$ ).

We will prove this by showing as loop invariant that at each stage there is an order- $p$ automorphism of $G$ that contains $\mathcal{C}$ among its $p$-cycle set and contains $X$ in its fixed point set. Clearly, before the loop is entered, there is an order- $p$ automorphism of $G_{[X]}$ with $\mathcal{C}=\emptyset$ as subset of its $p$-cycle set. Suppose this property holds at the beginning of some iteration of the for-loop. Suppose in the next iteration a new $p$-cycle $C$ gets included in $\mathcal{C}$. We have to show that there is an order- $p$ automorphism of $G$ with $X$ as fixed-point set and such that $\mathcal{C} \cup\{C\}$ is contained in its $p$-cycle set. Consider $C y c l e\left(G_{[C]}^{\prime}, G_{[r(C)]}^{\prime \prime}, \ldots, G_{[r(C)]}^{\prime \prime}\right)$, which is in $m_{0} d_{p}$-GA. Notice that the corresponding order-p automorphism $\psi$ of $\operatorname{Cycle}\left(G_{[C]}^{\prime}, G_{[r(C)]}^{\prime \prime}, \ldots, G_{[r(C)]}^{\prime \prime}\right)$ cannot map the copy of $G_{[C]}^{\prime}$ to itself since $G_{[C]}^{\prime}$ cannot have order-p automorphisms (because it forces $G$ to have order-p automorphisms with $X \cup C$ as fixed points). Thus $\psi$ must map the $p$ graphs in $C y c l e\left(G_{[C]}^{\prime}, G_{[r(C)]}^{\prime \prime}, \ldots, G_{[r(C)]}^{\prime \prime}\right)$ by a $p$-cyclic rotation. In particular, it implies that $\psi$ maps $G_{[C]}^{\prime}$ to some copy of $G_{[r(C)]}^{\prime \prime}$. Hence, $\psi$ restricted to the nodes of $G$ yields an automorphism $\phi$ of $G$ with $X$ as fixed-point set and such that $\mathcal{C} \cup\{C\}$ is contained in the p-cycle set of $\phi$.

By induction, it follows that when the loop is exited we have an order- $p$ automorphism which is completely specified: $\mathcal{C}$ is its collection of $p$-cycles and $X$ is the fixed point set.

\section{Proof of Theorem 12}

We will prove the theorem by induction on $k$. Notice that the base case for $k=1$ is proven in Lemma 13. More precisely, the induction hypothesis is the following:

Suppose that we have a polynomial time algorithm $\mathcal{A}_{k-1}$ with oracle $\bmod _{p}$-GA that computes an order- $p^{k-1}$ subgroup of $A u t(G)$ given a graph $G \in \bmod _{p^{k-1}-\mathrm{GA}}$ as input.

We now prove the induction step by designing a polynomial-time algorithm with oracle $\bmod _{p^{-}}$GA that, given as input a graph $G \in \bmod _{p^{k}} \mathrm{GA}$, computes the elements of an order- $p^{k}$ 
subgroup of $A u t(G)$. The induction hypothesis gives us the algorithm $\mathcal{A}_{k-1}$ using which we can compute in polynomial time an order- $p^{k-1}$ subgroup of $A u t(G)$ (call it $\left.S_{k-1}\right)$. Let

$$
S_{k-1}=\left\{g_{1}, g_{2}, \ldots, g_{p^{k-1}}\right\}
$$

We first recall the following result from the theory of p-groups (for instance, it is wellknown consequence of Sylow's theorems) [7]:

Proposition 14 If $A$ is a finite group such that $p^{k}$ divides $|A|$ for some prime $p$, then for every subgroup $H$ of $A$ such that $|H|=p^{k-1}$ there exists a subgroup $K$ of $A$ such that $|K|=p^{k}$ and $H$ is a normal subgroup of $K$.

Let now $G$ be a graph such that $G \in \bmod _{p^{k}}$ GA. Furthermore, let $S_{k-1}$ be a subgroup of $\operatorname{Aut}(G)$ such that $\left|S_{k-1}\right|=p^{k-1}$. An immediate consequence of this proposition is the following fact.

Fact 15 There is a subgroup $S_{k}$ of $A u t(G)$ of order $p^{k}$ such that $S_{k-1}$ is a normal subgroup of $S_{k}$.

The quotient group $S_{k} / S_{k-1}$ has $p$ elements, more explicitly we can write it as

$$
S_{k} / S_{k-1}=\left\{S_{k-1} g, \ldots, S_{k-1} g^{p}=S_{k-1}\right\} \text {, for some } g \in S_{k}
$$

As a first step to designing the required algorithm for computing $S_{k}$ we prove the following claim.

Claim. $G \in \bmod _{p^{k}}$ GA iff there exists $g \in A u t(G)$ such that the following hold

1. $g \notin S_{k-1}$.

2. $o(g)=p^{l}$ for some $l \leq k$.

3. $S_{k-1} g=g S_{k-1}$

Proof. Clearly the forward direction of the claim is the fact stated above. To prove the reverse implication suppose there exists $g \in A u t(G)$ satisfying the above three conditions. Consider the group $H$ generated by the set $S_{k-1} \cup\{g\}$. Since $S_{k-1} g=g S_{k-1}$ it follows that $S_{k-1}$ is a normal subgroup of $H$. Notice that the quotient group $H / S_{k-1}$ is the cyclic group generated by $S_{k-1} g$ and therefore its order is a power of $p$ (more precisely, it is $p^{j}$ for some $j \leq l)$. Since $p$ must divide $\left|H / S_{k-1}\right|$ it follows that $p^{k}$ divides $|H|$ and thus it also divides $|A u t(G)|$ proving the claim.

Observation 16 Notice that if we compute an element $g$ described in the above claim, we can compute (in polynomial time, by brute-force listing) the subgroup $H$ generated by the set $S_{k-1} \cup\{g\}$. Applying Proposition 14 we know that there is a subgroup $S_{k}$ of $H$ such that $\left|S_{k}\right|=p^{k}$ and $S_{k-1}$ is normal in $S_{k}$. Since $H$ has at most $p^{2 k}$ elements, we can do a brute-force search for $S_{k}$ in polynomial time. 
It remains to show how, given an input graph $G \in \bmod _{p^{k}}$ GA and $S_{k-1}$, we can compute with a $\bmod _{p}$-GA oracle an element $g$ satisfying the properties of the above claim. Let

$$
S_{k-1}=\left\{g_{1}, g_{2}, \ldots, g_{p^{k-1}}\right\}
$$

For $1 \leq i \leq p^{k-1}$ let $F_{i}=\left\{j \in[n]: g_{i}(j)=j\right\}$ and $M_{i}=[n]-F_{i}$.

For simplicity we explain the rest of the algorithm in two phases. In the first phase of the algorithm we check if there is an automorphism of order $p^{l}$, for $l \leq k$, that fixes some $x_{i} \in M_{i}$ for all $i \in\left[p^{k}\right]$.

\section{Phase 1 of Algorithm $\mathcal{A}_{k}$ :}

for each choice $\left\{x_{i} \in M_{i}\right\}_{1 \leq i \leq p^{k-1}}$ do

$$
\text { for each } p \text {-cycle } P \in \bar{S}_{n} \text { disjoint from }\left\{x_{i}\right\}_{1 \leq i \leq p^{k-1}} \text { do }
$$

\{

$\mathcal{C} \leftarrow\left\{\left(x_{1}\right),\left(x_{2}\right), \ldots,\left(x_{p^{k-1}}\right), P\right\}$

$I^{*}$ Strictly speaking, in $\mathcal{C}$ there are no repetitions of the 1-cycles */

for each permutation $\tau$ of $\left[p^{k-1}\right]$ do

if $\operatorname{Comb}\left(\tau, G, S_{k-1}, \mathcal{C}, p\right) \in \bmod _{p}-\mathrm{GA}$ then

\{

Use Algorithm $\mathcal{A}_{1}$ of Lemma 13 to compute an

order-p automorphism $\nu$ of $\operatorname{Comb}\left(\tau, G, S_{k-1}, \mathcal{C}, p\right)$;

Applying Lemma 11, from $\nu$ we compute an

order- $p$ automorphism $\pi$ of $G$;

\}

\}

To see the correctness of Phase 1 let $\operatorname{Comb}\left(\tau, G, S_{k-1}, \mathcal{C}, p\right)=\operatorname{Cycle}(H, K, \ldots, K)$ as in Lemma 11, with $p-1$ copies of $K$, where $H$ and $K$ are appropriately defined. Suppose $\nu$ maps $H$ to itself and each copy of $K$ to itself. Then $\nu$ projected to $H$ gives an order- $p$ automorphism of $G$ that fixes all points in $\left\{x_{i}\right\}_{1 \leq i \leq p^{k-1}}$ as well as all points in $P$. On the other hand, if $\nu$ cyclically rotates the $p$ graphs $(H, K, \ldots, K)$ then by Lemma 11 we get an automorphism $\psi$ of $G$ that has a $p$-cycle $P$ and fixes all points in $\left\{x_{i}\right\}_{1 \leq i \leq p^{k-1}}$. We can easily compute the order $o(\psi)=p r$. If we choose $\pi=\psi^{r}$ we get the desired order-p automorphism of $G$.

Thus, in either case if Phase 1 succeeds it outputs an order- $p$ automorphism $\pi \notin S_{k-1}$ such that $\pi S_{k-1}=S_{k-1} \pi$. Given this element $\pi$ we can compute an order- $p^{k}$ subgroup of $\operatorname{Aut}(G)$ (which contains $S_{k-1}$ ) by a brute-force search for it in the group generated by $S_{k-1} \cup\{\pi\}$.

The algorithm goes to the second phase if the first phase does not succeed. In the second phase of the algorithm we check if there is an automorphism of $G$ of order $p^{l}$, for some $l \leq k$, that differs from all automorphisms in $S_{k-1}$. In this phase, the correctness relies on the fact that Phase 1 has not succeeded. 
At this point we introduce some notation. Let $M$ denote $M_{1} \times \cdots \times M_{p^{k-1}}$. Let $C_{1}, \ldots, C_{t}$ be a collection of $t$ cycles such that for $1 \leq i \leq t\left|C_{i}\right|=p^{e_{i}}$ and $0 \leq e_{i} \leq k$. We say that the above collection $C_{1}, \ldots, C_{t}$ of cycles is good w. r. t. $S \subseteq\left[p^{k-1}\right],\left(l_{1}, l_{2}, \ldots, l_{p^{k-1}}\right) \in M$, and $\tilde{f}=\left\{f_{j} \in F_{j}: j \in S\right\}$ if

$$
\tilde{f} \subseteq \cup_{i=1}^{t} C_{i}, \text { and } l_{j} \in \cup_{i=1}^{t} C_{i} \text { for } 1 \leq j \leq p^{k-1}
$$

\section{Phase 2 of Algorithm $\mathcal{A}_{k}$ :}

for each $S \subseteq\left[p^{k-1}\right]$ do

for each tuple $\tilde{l}=\left(l_{1}, l_{2}, \ldots, l_{p^{k-1}}\right) \in M$ do

for each set $\tilde{f}=\left\{f_{j} \in F_{j}: j \in S\right\}$ do

for $t=1$ to $p^{k-1}$ do

for each cycle collection $\left\{C_{1}, \ldots, C_{t}\right\}$ that is $\operatorname{good}$ w. r. t. $S, \tilde{l}$, and $\tilde{f}$ do

\{

For each $j \in S$ check that $C_{1} C_{2} \cdots C_{t}\left(l_{j}\right)=g_{j}\left(l_{j}\right)$;

For each $j \notin S$ check that $C_{1} C_{2} \cdots C_{t}\left(l_{j}\right) \neq g_{j}\left(l_{j}\right)$;

For each $j \in S$ check that $C_{1} C_{2} \cdots C_{t}$ does not fix $f_{j}$;

if all the above three checks succeed then

\{

$$
\begin{aligned}
& \mathcal{C} \leftarrow\left\{C_{1}, \ldots, C_{t}\right\} \\
& \text { for each permutation } \tau \in S_{p^{k-1}} \text { do } \\
& \text { if } \operatorname{Comb}\left(\tau, G, S_{k-1}, \mathcal{C}, p\right) \in \bmod _{p} \text {-GA then } \\
& \{
\end{aligned}
$$

$I^{*}$ At this point there is $g \in A u t(G)$ of order $p^{l}$ that satisfies conditions of the Claim */ Construct such an automorphism $g$ by adaptively querying $\bmod _{p}$-GA for $\operatorname{Comb}\left(\tau, G, S_{k-1},\{C\} \cup \mathcal{C}, p\right)$

for different cycles $C$ of size $p^{\alpha}$ for $\alpha \leq k$, and including $C$ in $\mathcal{C}$ if $\operatorname{Comb}\left(\tau, G, S_{k-1},\{C\} \cup \mathcal{C}, p\right) \in \bmod _{p^{-}} \mathrm{GA}$;

if the above construction succeeds then output a desired automorphism $g$ of $G$ and stop

To see the correctness, we use the fact that the algorithm enters Phase 2 only if Phase 1 is completed unsuccessfully. Now, if $\operatorname{Comb}\left(\tau, G, S_{k-1}, \mathcal{C}, p\right) \in \bmod _{p}$ GA it is not possible that the witnessing order-p automorphism of $\operatorname{Comb}\left(\tau, G, S_{k-1}, \mathcal{C}, p\right)=\operatorname{Cycle}(H, K, \ldots, K)$ maps the copy of $H$ and each copy of $K$ to themselves. Otherwise we would have an order- $p^{l}$ (for some $l \leq k$ ) automorphism of $G$ that commutes with $S_{k-1}$ and fixes each point in a collection $l_{i} \in M_{i}, 1 \leq i \leq p^{k-1}$, contradicting Phase 1's failure. Thus, it follows that the order-p automorphism of $\operatorname{Comb}\left(\tau, G, S_{k-1}, \mathcal{C}, p\right)$ must cyclically permute the copy of $H$ and $p-1$ copies of $K$. Hence $C_{1}, \ldots, C_{t}$ are cycles of the corresponding order- $p^{l}$ automorphism 
of $G$, which is computed in the last step of the algorithm. Observe that the three checks made in Phase 2 guarantee that the sought for automorphism with $C_{1}, \ldots, C_{t}$ as a subset of its cycles is not in $S_{k-1}$. Now, it is not hard to see that if $p^{k}$ divides $|A u t(G)|$ then, an element $g$ promised by the Claim is computed either in Phase 1 or in Phase 2.

We can compute an order- $p^{k}$ subgroup of $A u t(G)$ (which contains $S_{k-1}$ ) by a brute-force search for it in the group generated by $S_{k-1} \cup\{g\}$.

Notice the following immediate consequence of Theorem 12. Interestingly, it is analogous to the well-known result that $\operatorname{Mod}_{p} \mathrm{P}$ and $\operatorname{Mod}_{p^{k}} \mathrm{P}$ are identical. However, technically the proof of Theorem 12 is very different in nature.

Corollary 17 For any prime $p$ and any $k>0, \bmod _{p^{-}} \mathrm{GA}$ and $\bmod _{p^{k}}$ GA are polynomialtime Turing equivalent.

Another consequence of Theorem 12 is that search is polynomial-time Turing reducible to decision for $\bmod _{k}$-GA, for a search problem such as the one defined at the beginning of this section.

Corollary 18 For each $k>1$, search is polynomial-time Turing reducible to decision for $\bmod _{k}-\mathrm{GA}$.

\section{A Program Checker for $\bmod _{k^{-}} \mathrm{GA}$}

The goal of this section is to show that for each $k>1$ the decision problem mod $_{k}$-GA has a program checker in the sense of [3]. We first recall the definition of program checkers.

Definition 19 [3] A program checker $C_{A}$ for a decision problem $A$ is a (probabilistic) algorithm that for any program $P$ (supposedly for $A$ ) that halts on all instances, for any instance $x_{0}$ of $A$, and for any positive integer $k$ (the security parameter) presented in unary:

1. If $P$ is a correct program, that is, if $P(x)=A(x)$ for all instances $x$, then with probability $\geq 1-2^{-k}, C_{A}\left(x_{0}, P, k\right)=$ Correct.

2. If $P\left(x_{0}\right) \neq A\left(x_{0}\right)$ then with probability $\geq 1-2^{-k}, C_{A}\left(x_{0}, P, k\right)=$ Incorrect.

The probability is computed over the sequences of coin flips that $C_{A}$ could have tossed. Also $C_{A}$ is allowed to make queries to the program $P$ on some instances.

Before we proceed we also need the definition of IP protocols which was first introduced in $[6]$.

Definition 20 An interactive proof system consists of a prover-verifier pair $P \leftrightarrow V$. The verifier $V$ is a probabilistic polynomial time machine and the prover $P$ is, in general, a machine of unlimited computational power which shares the input tape and a communication tape with $V$.

$P \leftrightarrow V$ is an interactive (i.e. IP) protocol for a language $L$, if for every $x \in \Sigma^{*}$ :

$$
\begin{aligned}
& x \in L \rightarrow \operatorname{Prob}[P \text { makes } V \text { accept }]>3 / 4, \\
& x \notin L \rightarrow \forall \text { provers } P^{\prime}: \operatorname{Prob}\left[P^{\prime} \text { makes } V \text { accept }\right]<1 / 4,
\end{aligned}
$$


The design of our checker for $\bmod _{k}-\mathrm{GA}$ is based on the following theorem [3].

Theorem 21 [3] If a decision problem A and its complement have both interactive proof systems, in each of which the honest prover can be simulated in polynomial time with queries to $A$, then $A$ has a polynomial-time program checker.

We will first provide a program checker for $\bmod _{p}-\mathrm{GA}$, for any prime $p$. Notice that Lemma 13 already gives an IP protocol for $\bmod _{p}$-GA with the prover polynomial-time Turing

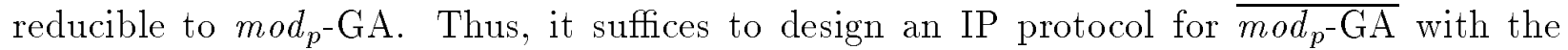
requisite properties.

Lemma 22 For any prime $p$, there is an IP protocol for $\overline{\bmod _{p}-\mathrm{GA}}$ in which the honest prover is polynomial-time Turing reducible to $\bmod _{p}-\mathrm{GA}$.

Proof. We rewrite the definition of $\overline{\bmod _{p}-\mathrm{GA}}$ as follows: $\overline{\bmod _{p}-\mathrm{GA}}=\{G: G$ has no automorphism with a $p$-cycle $\}$. Given an input graph $G$, the aim is to design an IP protocol which accepts $G$ with high probability if $G$ has no automorphism with a $p$-cycle, and which rejects $G$ with high probability otherwise. Notice that since the prime $p$ is a constant, the total number of $p$-cycles in $S_{n}$ is bounded by $q n^{p}$, where $q$ is a constant. We will build the desired IP protocol from an IP protocol for the following related language $L=\{(G, C)$ : $|V(G)|=n, C \in S_{n}$ is a $p$-cycle and $G$ has no automorphism with $C$ as one of its cycles $\}$.

\section{2-round IP Protocol for $L$ :}

input $(G, C)$;

$Y \leftarrow[n]-\{i: i \in C\}$

\section{Verifier:}

Pick a permutation $\psi \in S_{Y}$ uniformly at random;

Pick a random bit $b \in\{0,1\}$;

if $b=0$ then

else

$$
\text { send } G^{\prime}=\psi(G) \text { to the Prover }
$$

$$
\text { send } G^{\prime}=\psi \circ C(G) \text { to the Prover }
$$

\section{Prover:}

if there exists permutation $\pi \in S_{Y}$ such that $\pi(G)=G^{\prime}$ then

send back a bit $c=0$

else

send back a bit $c=1$

if $c=b$ then

Verifier accepts

else

Verifier rejects

We first show that if the prover is honest then the protocol accepts an input $(G, C) \in L$ with probability 1 . Suppose $b$ took the value 0 and the graph $\psi(G)=G^{\prime}$ was sent to the 
prover. Then clearly, the prover will find a permutation, namely $\psi$, such that $\psi(G)=G^{\prime}$ and send back $c=0$ leading to the acceptance of the input. Next, suppose $b$ took the value 1 . In that case we claim that there does not exist any permutation $\pi \in S_{Y}$ such that $\pi(G)=G^{\prime}$. Suppose there exists such a $\pi$. Then, since $\pi(G)=\psi \circ C(G)$, it follows that $(\pi)^{-1} \psi \circ C$ is in $\operatorname{Aut}(G)$, which contradicts the assumption that $(G, C) \in L$. In this case the prover will send back $c=1$ and the verifier will again accept.

Now, to prove the soundness of the protocol, we must show that for an input $(G, C) \notin L$, the verifier will reject the input with probability at least $1 / 2$, for any prover. We first need the following claim. In the sequel we use $X$ to denote the set $\{i: i \in C\}$ and $Y$ to denote $[n]-X$.

Claim A. If $G$ has an automorphism $\tau$ with $C$ as one of its cycles then the random graphs $\psi(G)$ and $\psi \circ C(G)$ are identically distributed, where $\psi$ is picked uniformly at random from $S_{Y}$.

Proof. Let $\tau=\rho \circ C$, where $\rho \in S_{Y}$. From $\rho \circ C(G)=G$ it is not hard to see that for any graph $H$

$$
\exists \alpha \in S_{Y}[\alpha(G)=H] \Longleftrightarrow \exists \beta \in S_{Y}[\beta \circ C(G)=H]
$$

Thus for any graph $H$

$$
\operatorname{Prob}_{\alpha}[\alpha(G)=H]=0 \Longleftrightarrow \operatorname{Prob}_{\beta}[\beta \circ C(G)=H]=0
$$

where $\alpha$ and $\beta$ are picked uniformly at random from $S_{Y}$.

Now, since $\alpha(G)=H$ iff $\alpha \rho \circ C(G)=H$, it is straightforward to derive that the set of permutations $\left\{\beta \in S_{Y}: \beta \circ C(G)=H\right\}$ is precisely $\varphi A u t\left(G_{[X]}\right) \rho$ which is of size $\left|A u t\left(G_{[X]}\right)\right|$. Therefore,

$$
\operatorname{Prob}_{\beta}[\beta \circ C(G)=H]=\left|A u t\left(G_{[X]}\right)\right| /(n-p) !
$$

where $\beta$ is picked uniformly at random from $S_{Y}$.

It follows from Claim A that if $(G, C) \notin L$ the prover cannot distinguish between whether $G^{\prime}$ came from the case $b=0$ or from $b=1$. In fact, whether $b=0$ or $b=1$ the prover will find a $\pi \in S_{Y}$ such that $\pi(G)=G^{\prime}$. Therefore, the bit $c$ that is sent back by any (even cheating) prover can agree with $b$ with probability at most $1 / 2$. Consequently, the verifier will reject an input $(G, C) \notin L$ with probability at least $1 / 2$. We now describe the IP protocol for $\overline{\bmod _{p}-\mathrm{GA}}$.

IP Protocol for $\overline{\bmod _{p}-\mathrm{GA}}$ :

input $G ; /^{*} G$ has $n$ nodes */

bool $\leftarrow$ true;

for each $p$-cycle $C \in S_{n}$ do

if the IP protocol for $L$ rejects $(G, C)$ then bool $\leftarrow$ false;

if bool=true then Verifier accepts else Verifier rejects 
Since $G \in \overline{\bmod _{p} \text {-GA }}$ iff $(G, C) \in L$ for every $p$-cycle $C$, and since the IP protocol for $L$ has one-sided error it easily follows that the above IP protocol accepts $G \in \overline{\bmod _{p} \text {-GA }}$ with probability 1 and rejects $G \in \bmod _{p}$-GA with probability at least $1 / 2$. The error probability can be made exponentially small (say $2^{-n}$ ) in the above protocol by repeating the protocol ${ }^{4}$ (in parallel or sequentially).

The following claim completes the proof of the lemma.

Claim B. There is an honest prover that is polynomial-time Turing reducible to mod $_{p}$-GA for the above IP protocol for $\overline{\bmod _{p}-\mathrm{GA}}$.

Proof. First we observe that in bounding the complexity of the honest prover we are concerned about inputs $G \in \overline{\bmod _{p}-\mathrm{GA}}$. More precisely, we must show that there is a polynomial-time algorithm with $\bmod _{p}-\mathrm{GA}$ as oracle that can simulate the honest prover correctly for inputs $G \in \overline{\bmod _{p}-\mathrm{GA}}$. Notice that the honest prover of the overall IP protocol must actually simulate the honest prover of the IP protocol for $L$ for each input in the set

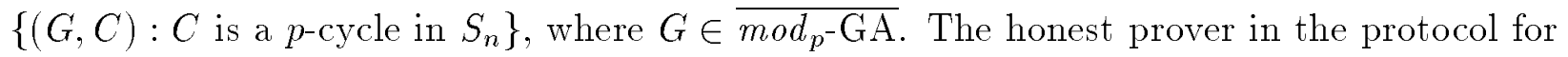
$L$ is supposed to try and compute a permutation $\pi \in S_{Y}$ such that $\pi(G)=G^{\prime}$. We have already argued in the correctness proof that for $G \in \overline{\bmod _{p} \text {-GA }}$ such a permutation $\pi$ exists if and only if the outcome of $b$ is 0 and $G^{\prime}=\psi(G)$ for the random permutation $\psi \in S_{Y}$. The honest prover constructs the graph $G^{\prime \prime}=C y c l e\left(G_{[X]}, G_{[X]}^{\prime}, \ldots, G_{[X]}^{\prime}\right)$, with $p-1$ copies of $G_{[X]}^{\prime}$. Using algorithm $\mathcal{A}_{1}$ of Lemma 13 the honest prover computes an automorphism of $G^{\prime \prime}$ of order $p$ if it exists. Notice that if there is a permutation $\pi \in S_{Y}$ such that $\pi(G)=G^{\prime}$ then there is a permutation $\pi^{\prime}$ such that $\pi^{\prime}\left(G_{[X]}\right)=G_{[X]}^{\prime}$. Hence we can find an order- $p$ automorphism of $G^{\prime \prime}$ which cyclically permutes the $p$ graphs in $G^{\prime \prime}$, by mapping the copy of $G_{[X]}$ to the first copy of $G_{[X]}^{\prime}$ by $\pi^{\prime}$, and each of the first $p-2$ copies of $G_{[X]}^{\prime}$ are mapped to the next copy of $G_{[X]}^{\prime}$ by the identity permutation, and finally, the last copy of $G_{[X]}^{\prime}$ is mapped back to $G_{[X]}$ by $\pi^{\prime-1}$. It is easy to see that this is an automorphism of $G^{\prime \prime}$ of order $p$. Conversely, suppose that $G^{\prime \prime}$ has an order- $p$ automorphism $\tau$ computed by the honest prover. Since $G \notin \bmod _{p}-\mathrm{GA}$ and $G^{\prime} \notin \bmod _{p}-\mathrm{GA}$, the $p$ graphs defining $G^{\prime \prime}$ must be rotated in some $p$-cyclic order by the automorphism $\tau$. It follows that the copy of $G_{[X]}$ is mapped by $\tau$ to some copy of $G_{[X]}^{\prime}$. Let $\pi^{\prime}$ be the projection of $\tau$ to these two copies. We have $\pi^{\prime}\left(G_{[X]}\right)=G_{[X]}^{\prime}$. From $\pi^{\prime}$ we can easily recover a permutation $\pi \in S_{Y}$ such that $\pi(G)=G^{\prime}$. Thus the honest prover finds an order-p automorphism $\tau$ of $G^{\prime \prime}$ iff there exists $\pi \in S_{Y}$ such that $\pi(G)=G^{\prime}$, and moreover, from such a $\tau$ the corresponding $\pi$ is easily computed. Hence, the honest prover is polynomial-time Turing reducible to $\bmod _{p}$-GA.

We can now conclude that, for any prime $p, \bmod _{p}$-GA has an efficient program checker.

Theorem 23 For any prime $p$, mod $d_{p}$ GA has a polynomial-time program checker.

Proof. Note that from Lemma 13 we get an IP protocol for $\bmod _{p}$-GA with the prover polynomial-time Turing reducible to $\bmod _{p}$-GA and that by Lemma 22 an IP protocol with

\footnotetext{
${ }^{4}$ With some modifications we can easily get a constant round IP protocol.
} 
requisite properties exists for $\overline{\bmod _{p}-\mathrm{GA}}$. Now, Theorem 21 proves the existence of an efficient checker for $\bmod _{k}-\mathrm{GA}$.

Now it is easy to provide a checker for any $\bmod _{k}-\mathrm{GA}$ problem.

Theorem 24 For each $k>1, \bmod _{k}$-GA has a polynomial-time program checker.

Proof. Let $\prod_{1 \leq i \leq m} p_{i}^{e_{i}}$ be the prime factorization of $k$. Because the class of checkable sets is obviously closed under join and under Turing equivalence [3], by Theorem 23 it suffices to show that $\bmod _{k}-\mathrm{GA} \equiv_{T}^{p} \bmod _{p_{1}}-\mathrm{GA} \oplus \cdots \oplus \bmod _{p_{m}}-\mathrm{GA}$. Observe that a graph $G$ belongs to

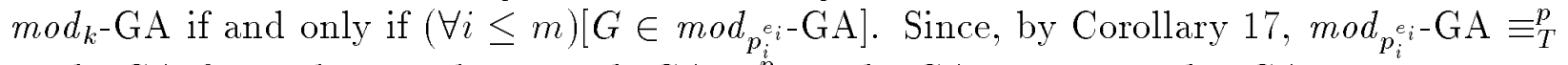
$\bmod _{p_{i}}$ GA for each $i$, we have $\bmod _{k}$-GA $\leq_{T}^{i} \bmod _{p_{1}}-\mathrm{GA} \oplus \cdots \oplus \bmod _{p_{m}}$-GA. It is easy to prove that $\bmod _{p_{i}}-\mathrm{GA} \leq_{T}^{p} \bmod _{k}$-GA for each $i$. Therefore, $\bmod _{p_{1}}-\mathrm{GA} \oplus \cdots \oplus \bmod _{p_{m}}-\mathrm{GA} \leq_{T}^{p}$ $\bmod _{k}-\mathrm{GA}$ as well.

\section{Concluding Remarks}

In this paper we define modular graph automorphism problems $\left(\bmod _{k}-\mathrm{GA}\right)$ and locate them between GA and GI. We also design an efficient program checker for $\bmod _{k}$-GA based on an algorithm that reduces search to decision for $\bmod _{k}$-GA and an IP protocol for $\overline{\bmod _{k} \text {-GA. }}$ The bottleneck in making our checker nonadaptive is essentially the following: can search be reduced to decision via parallel queries for $\bmod _{p}$-GA, for prime $p$ ?

Indeed, our initial motivation in studying the $\bmod _{k}$-GA problems was to understand the difference between GI and GA by introducing problems of intermediate difficulty. In this context, a challenging question is whether search reduces to decision via parallel queries for GI (hence yielding nonadaptive checkers for GI). We believe that as a first step this question must be answered for $\bmod _{p}$-GA.

\section{References}

[1] J. Balcázar, J. Díaz, and J. Gabarró. Structural Complexity II. Springer-Verlag, 1990.

[2] J. Balcázar, J. Díaz, and J. Gabarró. Structural Complexity I. Springer-Verlag, second edition, 1995.

[3] M. Blum and S. Kannan. Designing programs that check their work, Journal of the ACM, 43:269-291, 1995.

[4] M. Blum, M. M. Luby, and R. Rubinfeld. Self-testing/correcting with applications to numerical problems, J. Comput. Syst. Sci. 47:73-83, 1993.

[5] R. Chang. On the structure of NP computations under boolean operators. Ph. D. Thesis, Cornell University, 1991. 
[6] S. Goldwasser, S. Micali, and C. Rackoff. The knowledge complexity of interactive proof systems. SIAM Journal on Computing 18:186-208, 1989.

[7] M. Hall. The Theory of Groups, Macmillan, New York, 1959.

[8] F. Harary. Graph Theory, Addison Wesley, Reading, 1969.

[9] C. Hoffman. Subcomplete generalizations of graph isomorphism. Journal of Computer and System Sciences, 25:332-359, 1982.

[10] J. Köbler, U. Schöning, and J. Torán. The graph isomorphism problem: its structural complexity, Birkhäuser, Boston, 1993.

[11] A. Lozano and J. Torán. On the nonuniform complexity of the graph isomorphism problem. In Proceedings of the 7th Structure in Complexity Theory Conference, pp. 118$129,1992$.

[12] R. Mathon. A note on the graph isomorphism counting problem. Information Processing Letters, 8:131-132, 1979.

[13] C. P. Schnorr. On self-transformable combinatorial problems. Math. Programming Study, 14:95-103, 1982. 